\title{
Year One Progress Report Three-dimensional Imaging of Drill Core Samples using Synchrotoron Computed Microtomography
}

\author{
Brent Lindquist - Principle Investigator \\ David Coker \\ Sangmoon Lee
}

State University of New York, Stony Brook, NY 11794-3600

\section{Introduction}

Fundamental theories of rock structure are limited by the absence of high resolution, pore level, three dimensional images which could be used for statistical analysis. The ability to produce such images in a non-destructive manner would also allow for repeated measurements of dynamic processes such as fluid motion which could be correlated to the medium properties.

One aspect of this funded work is the production of cross sectional images of rock drill core sampies with one micron resolution. This work is being done by Keith Jones and Per Spanne of Brookhaven National Laboratory using beam lines X-17 and X-26 of the National Synchrotron Light Source. Jones's annual report will provide a description of the year one progress in this area.

The second aspect of this work is the topological and statistical analysis of the pore structure of the three dimensional images provided by our BNL collaborators. We report here on the progress in this area that has been made in year one. Year one effort has focused on the problem of definition of pore space in any given slice (image filtering), analysis of the filtered images via 2-point correlation st,-ucture, and on the development of software to trace the connectivity of pores throughout the cross sectional images.

The report is divided into the following sections: $\$ 2$ - a description of the 3D data sets that have been provided by our BNL collaborators; $§ 3$ data filtering; $\S 4$ - 2-point correlation function analysis; $§ 5$ - development of connectivity tracing software; and $\S 6$ - proposed work for year two.

\section{DISCLAIMER}

This report was prepared as an account of work sponsored by an agency of the United States Government. Neither the United States Government nor any agency thereof, nor any of their employees, makes any warranty, express or implied, or assumes any legal liability or responsibility for the accuracy, completeness, or usefulness of any information, apparatus, product, or brift lor its use would not infringe privately owned rights. Reference herein to any specific commercial product, process, or service by trade name, trademark, ence herein to any secture not necessarily constitute or imply its endorsement, recom mendation, or favoring by the United States Government or any agency thereof. The views and opinions of authors expressed herein do not necessarily state or reflect those of the United States Government or any agency thereof.

DISTRIBUTION OF THIS DOCUMENT IS UNLIMUTED 


\section{Rock Core Data Sets}

Five data sets of three dimensional rock core images have been provided for analysis. The data sets are summarized in Table 1. The glass bead sample provides a realization of a non-overlapping sphere model, a model which has been extensively studied. This sample thus provides a useful data set for debugging numerical software in development.

\begin{tabular}{|l|c|c|c|c|}
\hline rocik type & voxels/slice & voxel resolution & slices & slice separation \\
\hline $\begin{array}{l}\text { Berea Sandstone } \\
\text { (low permeability) }\end{array}$ & $501 \times 501$ & $2 \mu \mathrm{m}$ & 50 & $2 \mu \mathrm{m}$ \\
\hline $\begin{array}{l}\text { Berea Sandstone } \\
\text { (high permeability) }\end{array}$ & $541 \times 541$ & $5 \mu \mathrm{m}$ & 56 & $5 \mu \mathrm{m}$ \\
\hline Danish Chalk & $401 \times 401$ & $5 \mu \mathrm{m}$ & 22 & $5 \mu \mathrm{m}$ \\
\hline 100 micron glass beads & $601 \times 601$ & $5 \mu \mathrm{m}$ & 33 & $5 \mu \mathrm{m}$ \\
\hline Sandia drill cores & $600 \times 600$ & $50 \mu \mathrm{m}$ & 60 & $50 \mu \mathrm{m}$ \\
\hline
\end{tabular}

Table 1: Sumrnary of year one BNL data sets

\section{Data Filtering}

For quantitative analyses of the geologic samples obtained from X-ray tomography, it is necessary to have a well defined, self consistent method of specifying void space (pores) and material. Due to the nature of X-ray tomography, the distinction between void space and material is not well defined. The attenuation histogram for a sample consists of an overlapping bivariate distribution. (Figure 1 presents a typical histogram for a slice of the high porosity berea sandstone.) The peak at higher attenuation values is associated with material while the peak at lower attenuation values is associated with void space. Separation of these two overlapping distributions is an illposed problem and presents rnany difficulties with no unique solution. The traditional approach involves the choice of a cut-off attenuation value lying somewhere between the peaks. All voxels having attenuation values higher than this cutoff are then assumed to be material while voxels with smaller 


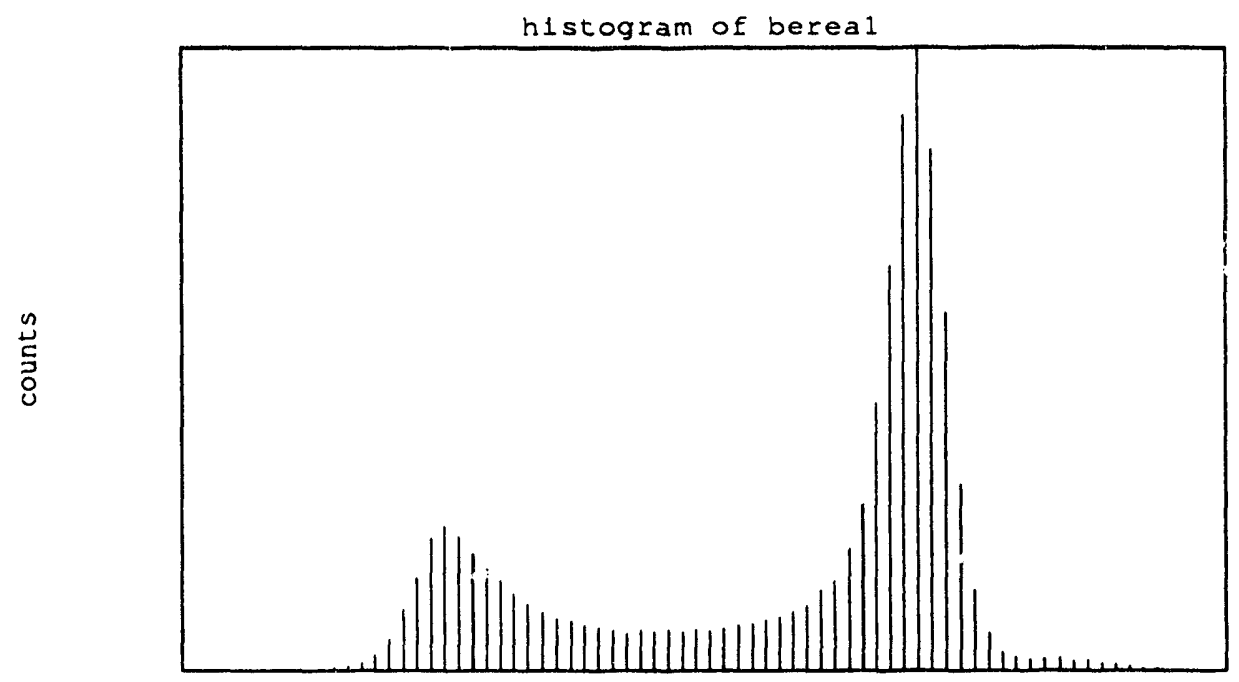

at tenuation

Figure 1: Example attenuation histogram (unfiltered) for high porosity berea sandstone.

attenuation values are assumed to be pore space. Choice of this cutoff is arbitrary and has little physical basis. To avoid this arbitrariness we have developed a consistent, statistical method for determining whether a voxel belongs to pore space or material.

Each peak is assumed to be Gaussian in nature. A Gaussian is fit to the material peak of the bivariate distribution using only the values on the high attenuation side of that peak. An example fit (solid line) to the material peak of Figure 1 is displayed in Figure 2. Any voxel having attenuation value greater than the mean value for the material peak is assumed to be a material voxel. For any attenuation value less than, but within 3 standard deviations of, the material peak mean value, the ratio of counts under the Gaussian fit to counts in the sample histogram is computed. For any voxel having this attenuation value, a random number (generated by a flat random number algorithm) in the range $(0,1)$ is chosen. If the random number value is less than the computed ratio for this attenuation value, the voxel is considered a material voxel. This procedure provides a statistical way of identifying the appropriate number of voxels corresponding to material in this range of 


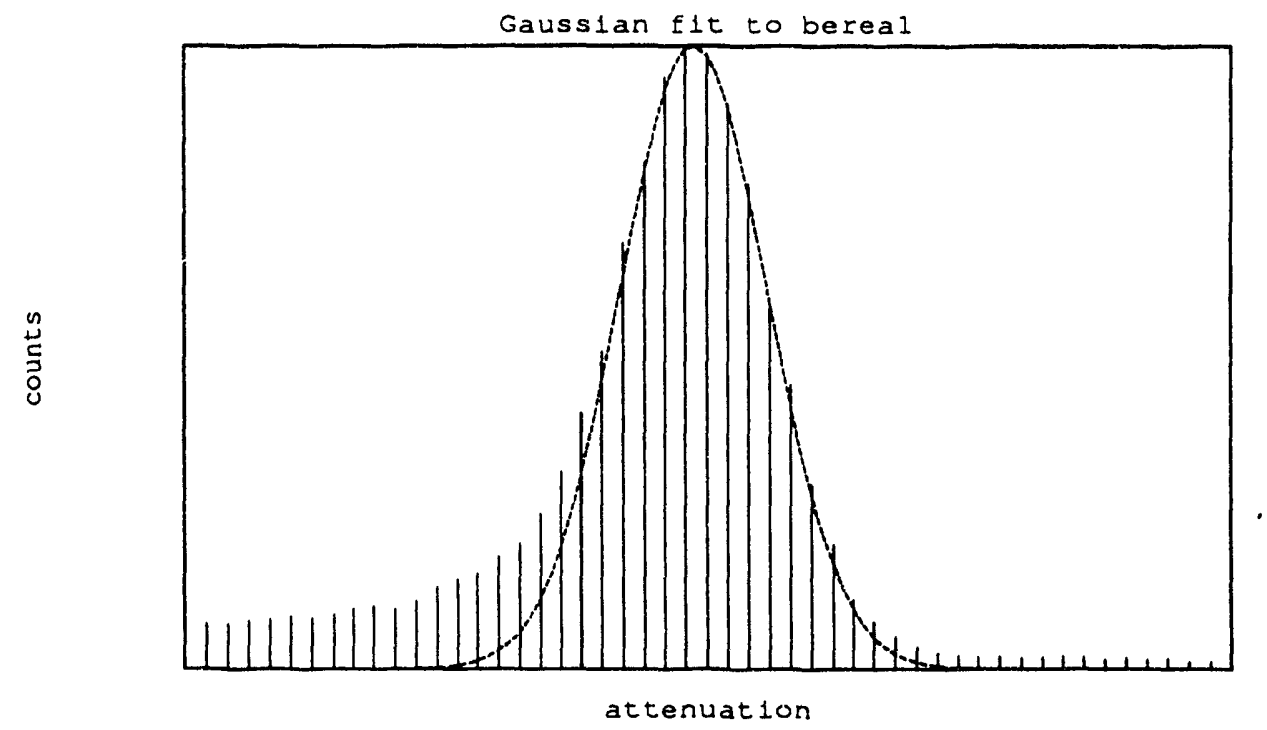

Figure 2: Gaussian fit (solid line) to the material peak of Figure 1.

attenuation values. This systematic procedure will slightly underestimate the material volume of the sample, as is demonstrated in the following discussion.

A Gaussian fit (using however only the values on the low attenuation side of the peak to determine the fit) can also be performed on the void peak in the bimodal distribution. Voxels having attenuation values lying below the mean value of the fitted peak are assumed to be void space. Voxels corresponding to attenuation values greater than, but within three standard deviations of, the mean peak value are filtered as above using a random number generator, to identify a statistically correct number of void space voxels in this attenuation range.

Together, these two fits identify a set of voxels as being either void space or material. The remaining voxels (identified by the random number generation scheme) lying in the "valley" between the peaks of the bimodal distribution largely correspond to voxels associated with the edges of individual granules in the sample. In practice, we identify this pool of remaining voxels as void space, thus slightly underestimating the sample's material volume, as stated above.

This procedure has been successfully applied to two samples of greatly 


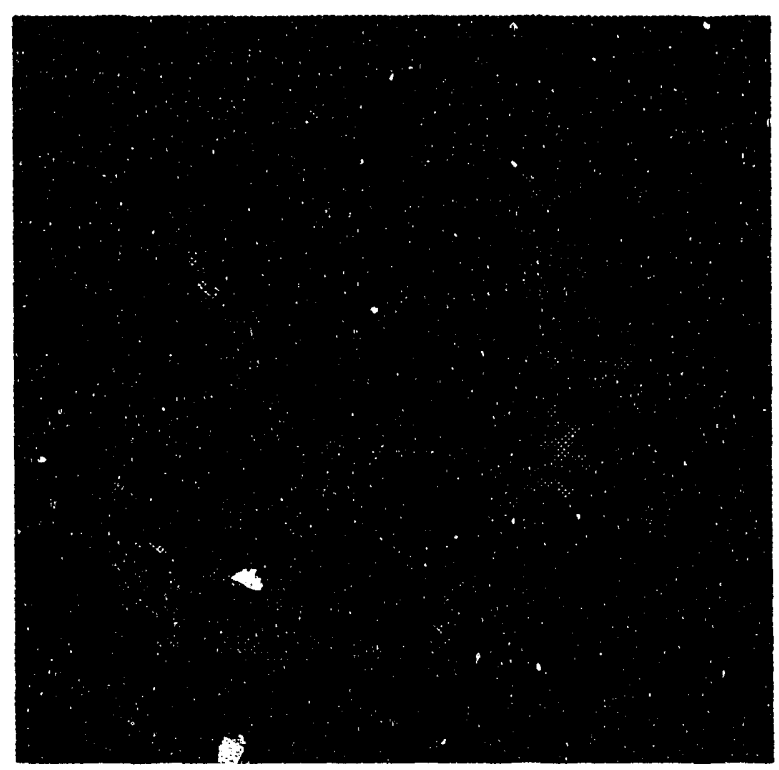

Figure 3: Unfiltered image of berea sandstone at imaged at $5 \mu \mathrm{m}$ per voxel.

different properties: a high porosity berea sandstone and a very low porosity sandstone featuring a crack. The final results indicate that our method is both efficient and useful. To demonstrate the result visually and to understand the net result from the above procedure, the prefiltered and postfiltered images of the berea sample are shown in Figures 3 and 4 respectively. The difference in contrast between the pre- and postfiltered images is visually apparent. In Figure 3, the edges of the individual granules are blurry and ill defined whereas those in Figure 4 are much more clearly defined.

This question of material/void identification is so inherent to the problem of working with the data sets that we plan further investigation in this area. We have just obtained software [27] capable of applying bivariate normal distribution analysis to the attenuation distributions, and we will be re-analyzing the data using fits obtained by this procedure. Further we are investigating the use of colored nose filtering to produce a cleaner image. 


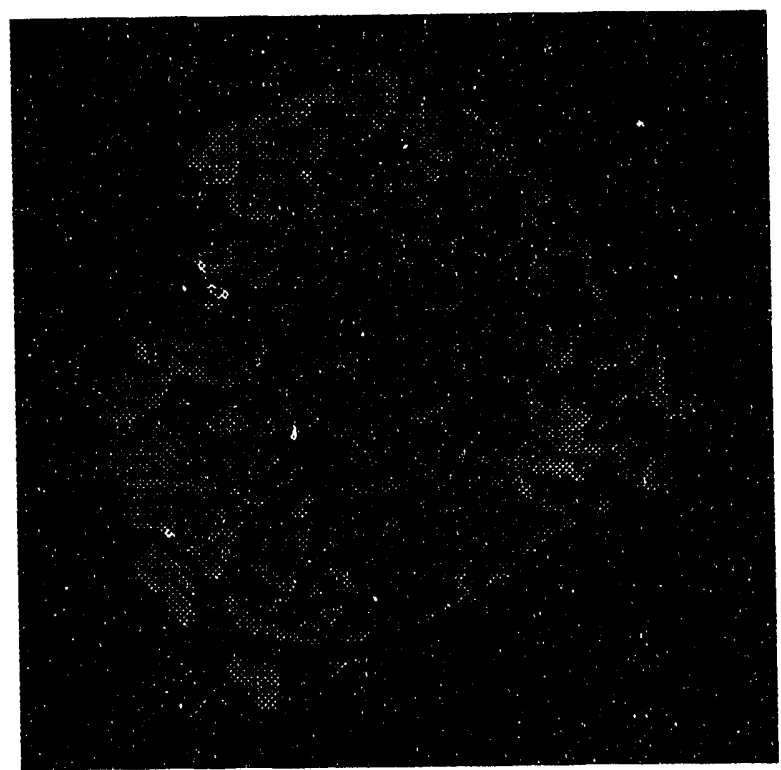

Figure 4: Filtered image of berea sandstone at imaged at $5 \mu \mathrm{m}$ per voxel. 


\section{Statistical Studies}

A body of theoretical work [1-13] exists relating the use of $n$-point correlation functions to obtain pore and particle size distributions and to predict material conductivities. Much of this theoretical work relates to overlapping and non-overlapping sphere models, for which exact solutions of the $n$-point correlation functions are available. Only a few papers in the literature address actual applications to physical samples. This is partly due to the computational expense of computing $n$-point correlation functions for $n>2$.

In inis past year, we have developed software, based upon the formulation of Berryman [1], for computing 2-point correlation functions for two dimensional slices.

The software handles a 2-dimensional sample, parts of which can be ignored in the analysis by defining a fiducial volume consisting of the union of non-overlapping polygons. This allows the void space exterior $i v$ the sample boundary, breakages, and other obvious imperfections in the sample slice to be ignored. Correlation functions can be calculated along two perpendicular directions for comparing anisotropy, or as a single isotropic average over the sample. The computational intensity has been addressed by porting the code to an Intel iPSC/860.

\begin{tabular}{|c|c|c|}
\hline$n$ & time (hr : min : sec) & $\epsilon$ \\
\hline 1 & $7: 23: 15$ & 1.000 \\
\hline 2 & $3: 42: 28$ & 0.996 \\
\hline 4 & $1: 51: 13$ & 0.996 \\
\hline 8 & $0: 56: 55$ & 0.974 \\
\hline 16 & $0: 29: 10$ & 0.950 \\
\hline 32 & $0: 15: 26$ & 0.898 \\
\hline
\end{tabular}

Table 2: Run times and parallelization efficiency for the correlation function code.

Table 2 shows run-times and parallelization efficiency for the isotropic 2-point correlation analysis of a $600 \times 600$ voxel sample (single polygon) as a function of the number, $n$, of processors. The parallelization efficiency is 
defined as

$$
\epsilon(n) \equiv \frac{t_{1}}{n t_{n}}
$$

where $t_{i}$ denotes the computational time required for $i$ nodes. The efficiency loss at large $n$ is due to the problem of load bala'scing amongst the processors. The current balancing algorithm assigns earh processor a unique set of separation values over which to compute correlation. It is clear that such a balancing algorithm decreases in efficiency as the number of processors approaches the total number of separations over which the correlation function is to be evaluated. An improved balancing scheme, which shares computation at a given separation amongst several processors will be implemented.

Using the filtered images, by assigning the material voxels a value of " 1 " and the void voxels a value of " 0 ", the 2-point correlation function $G_{2}(r)$ gives the probability that any pair of voxels separated by distance $r$ are both material voxels. We shall consider only the isotropic 2-point correlation calculation in this report.

The 2-point correlation function for the high porosity berea sample is shown in Figure 5. The computed porosity of the sample, obtained from the correlation function $y$-axis intercept is 0.37 , and the specific surface area, obtained from the initial slope is $2.7 \times 10^{4} \mathrm{~m}^{-1}$. The location of the first minima and the weak oscillations indicate an average granule size of $\sim 150 \mu \mathrm{m}$. This is consistent with the image in Figure 4.

Since attenuation is correlated with sample density, it is also possible to examine the homogeneity of the sample by density region. We proceed by dividing the material peak in the filtered image into six adjacent, one-standard deviation windows. By assigning all material voxels lying in any one chosen attenuation window the value " 1 ", and setting all remaining voxels to " 0 ", the 2-point correlation functions obtained for the six different windows can be compared with each other and with the full material computation. Due to the high resolution of these images $(2 \rightarrow 5 \mu \mathrm{m})$, this provides a high resolution probe for checking material density distributions that would otherwise be difficult to obtain. Fur the samples studied, it can be seen that there are indeed features in the rock that are brought out by this analysis. For exarnple, in the berea sample pictured in Figure 3, the high density material voxels exhibit more correlated structure than the low density material voxels as demonstrated by the existence of $10 \mu \mathrm{m}$ clumps in the low density ranges as compared with $20 \mu \mathrm{m}$ clumps in the high density ranges. This was not 


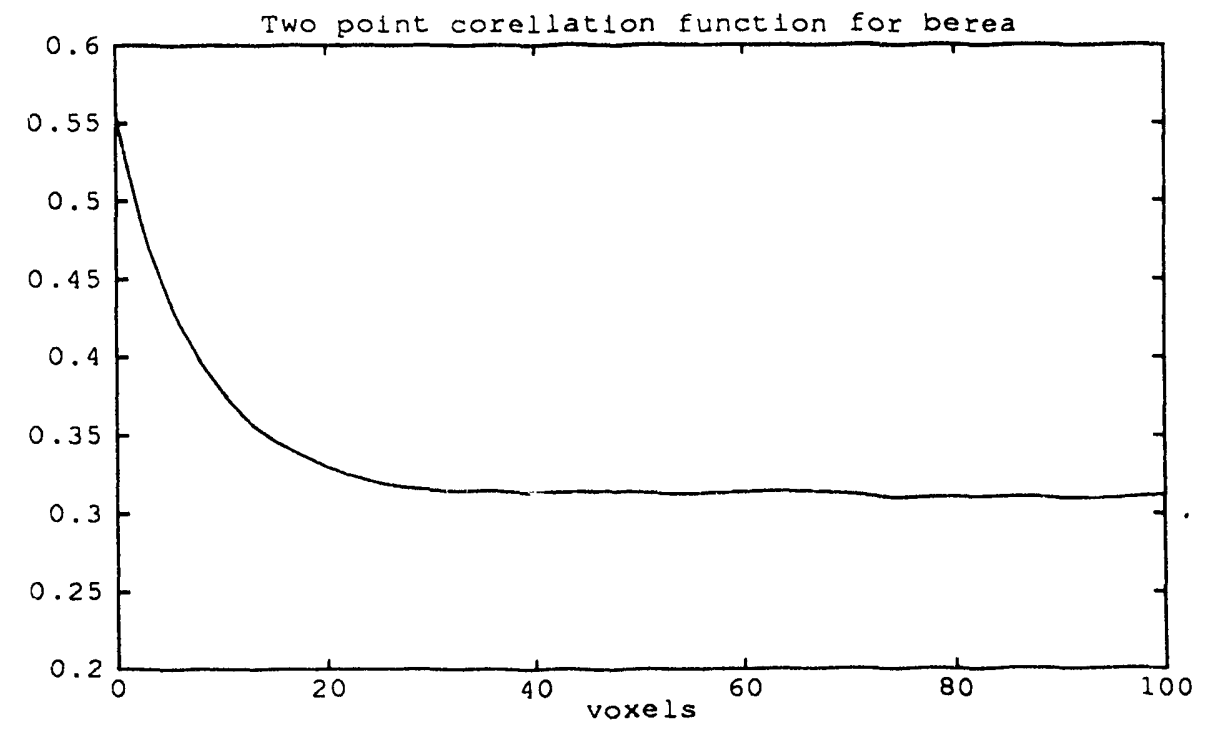

Figure 5: Example 2-point correlation function for berea. (1 voxel $=$ $5 \mu m)$.

apparent under visual inspection of the filtered images of these density regions. Thus, correlation studies provide not only structural information for the sample as a whole, but provide a means for understanding the nature and composition of the rock specimen. A more complete presentation of the filtration method and results is given in [22].

\section{Connectivity Work}

Although some work has been done in tracing pore connectivity through slices of porous material [14-19], they have been very labor intensive, involving a great deal of manual intervention, both in preparing slices and in analyzing them. Computer microtomography available and being developed at BNL allows for slice collection with minimal manual intervention (outside of sample set up and running software). We are in the process of developing software to handle the pore connectivity tracing with additional minimal manual intervention. Software has been completed which takes the filtered images of each slice and represents the void (equivalently material) spaces 
as a union of polygons. The question of mapping void spaces from one slice to its neighbors, and determining pore connectivity is ill determined since a method of interpolation between slices must be assumed. We are using the assumption that the spacing between slices is no worse than individual voxel size to guide the assumptions made in providing such interpolation. The pore tracing software will also contain a graphics component for visualization of the resultant images.

\section{Proposed Work - Year Two}

The ability to create three-dimensional images of the connective pore space opens up a wide range of possibilities for obtaining quantitative information regarding several geometric quantities of the pore structure. Of direct interest is the actual tortuosity of the three-dimensional sample. This factor describes the amount of winding or twisting that a fluid must undergo in traversing the material. Of direct application in predicting flow properties, the tortuosity is a free parameter in certain theoretical developments such as the commonly used Carmen-Kozeny relation. Direct measurement of this quantity in two-dimensions is somewhat difficult and unreliable. With three dimensional connectivity results, we will obtain more accurate measurements of the tortuosity distribution in a given rock sample. The three dimensional images will be analyzed for direct and reliable measurement of pore and pore-throat size distributions, which will have a resolution in the range of $2 \rightarrow 5 \mu m$.

One area of intense theoretical interest is the use of microscopic geometric information to predict flow properties. There have been several approaches this problem. We will explore the applicability of these methods to our high resolution data set as both a predictive tool and as a test of existing theories. The use of variational principles coupled with the use of $n$-point correlation functions is one such approach [3-5]. Unfortunately, previous attempts at obtaining these correlation functions have been limited by low resolution (optical methods) and poor reliability (destructive methods) of the data set. One of the open questions in this area is whether a real rock is better represented by a set of overlapping or nonoverlapping spheres. Our results seem to indicate that the latter is a better model for describing porous media. In the future, we propose to use 2-point correlation functions measured in both 
the two-dimensional slices and the full sample as inputs to these theories to predict permeabilities. The high resolution of the BNL data nakes our analysis unique. In addition to testing previous theories, we have some thoughts on improving previous variational results $[1,2,10]$ by incorporating additional terms.

Another manner of predicting flow properties is to run a single phase, fully saturated flow simulation, which produces a permeability prediction. Previous work [25] has been based mostly on simulated media (overlapping or nonoverlapping spheres) rather than actual media which somewhat limits their predictability. These models have, however, been useful in promoting further theoretical development by identifying pertinent predictive parameters. Simulation on real data samples would provide useful information for both predicting permeability of real media and improving existing models. Recently, we have been in contact with N. Martys [26] at NIST who has much experience with these type of simulations. He has expressed interest in collaborating with us in using the tomographic data as inputs for his simulations. The existing data sets in Table 1 do not provide enough depth to perform a three-dimensional analysis. However, it is hoped that our collaborators at Brookhaven will be able to provide such data sets in the future.

Recently, T.F. Wong, (Dept. of Geology, Stony Brook) has obtained some interesting results [24] using two-dimensional Scanning Electron Microscopy produced images to predict permeabilities using percolation models based on measuring chord length along arbitrary line segments. He has also expressed interest in a collaboration involving use of our data samples. The use of the tomographic data would allow him to automate his process and obtain a better understanding of chord distributions within a two-dimensional sample. Eventually, it would be desirable to extend this analysis to three-dimensions as well. An additional benefit would be the result that several theoretical methods for predicting permeability will have been applied to the same set of data samples.

\section{References}

[1] J.G. Berryman, J. Chem. Phys., 82, 1459-1467, (1985).

[2] J.G. Berryman, J. Chem. Phys., 83, 754-760, (1985). 
[3] S. Prager, Phys. Fluids, 4, 1477-1482, (1961).

[4] H.L. Weissberg and S. Prager, Phys. Fluids, 5, 1390-1392, (1962).

[5] H.L. Weissberg and S. Prager, Phys. Fluids, 13, 2958-2965, (1970).

[6] P. Debye, H.R. Anderson, and H. Brumberger, J. Appl. Phys., 28, 679-683, (1957).

[7] S. Torquato and G. Stell, J. Chem. Phys., 77, 2071-2077, (1982).

[8] S. Torquato and G. Stell, J. Chem. Phys., 78, 3262-3272, (1983).

[9] S. Torquato and G. Stell, J. Chem. Phys., 79, 1505-1510, (1983).

[10] M. Doi, J. Phys. Soc. Japan, 40, 567-572, (1976).

[11] J.G. Berryman, J. Appl. Phys., 57, 2374-2384, (1985).

[12] J.G. Berryman and S.C. Blair, J. Appl. Phys., 60, 1930-1938, (1986).

[13] J.G. Berryman and S.C. Blair, J. A. p. Phys., 62, 2221-2228, (1985).

[14] P.M. Kaufmann, F.A.L. Dullien, I.F. MacDonald, and C.S. Simpson, Acta Stereol. Suppl I, 2, 145-148, (1983).

[15] M. Yanuka, F.A.L. Dullien, D.E. Elrick, J. Microscopy, 135, 159-168, (1984).

[16] M. Yanuka, F.A.L. Dullien, D.E. Elrick, J. Coll. Interf. Sci., 112, 24$41,(1986)$.

[17] I.F. MacDonald, P.M. Kaufmann, and F.A.L. Dullien, J. Microscopy, 144, 277-296, (1986).

[18] M.J. Kwiecien, I.F. MacDonald, and F.A.L. Dullien, J. Microscopy, 159, 343-359, (1990).

[19] J.A. Quiblier, J. Coll. Interf. Sci., 98, 84-102, (1984).

[20] P.M. Adler, C.G. Jacquin, and J. Thovert, Water Res. Res., 28, 15711576, (1992). 
[21] A. Henriette, C.G. Jacquin, and P.M. Adler, Physico Chem. Hydro., 11, 63-80, (1989).

[22] D.A. Coker, K.W. Jones, W.B. Lindquist, and P. Spanne, document in preparation.

[23] R.A. Johns, J.S. Steude, L.M. Castanier, and P.V. Roberts, J. Geophys. Res., 98, 1889-1900, (1993).

[24] T.F. Wong, private communicition.

[25] N. Martys, Phys. Rev., B46, 61880-6090, (1992).

[26] N. Martys, private communication.

[27] H. C. Thode, S. J. Finch, and N. R. Menciell, Biometrics, 44, 11951201, (1988). 

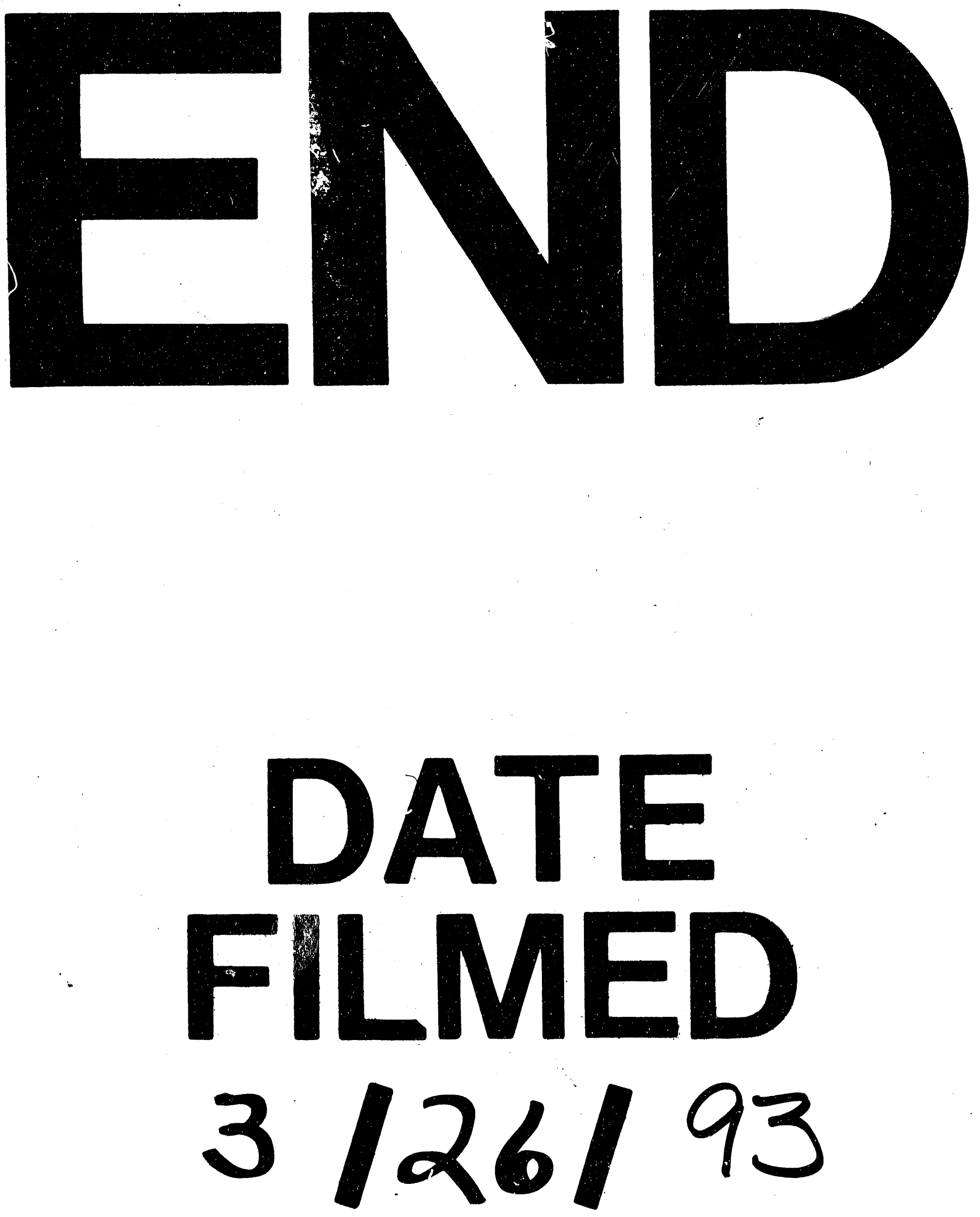
